\title{
Microsporidiose humana na síndrome de imunodeficiência adquirida
}

\author{
P. BRASIL, D. BONFIM DE LIMA, H. MOURA
}

Disciplinas de Doenças I nfecciosas e Parasitárias e de Parasitol ogia da Faculdade de Ciências Médicas do H ospital Universitário Pedro Ernesto, Universidade Estadual do Rio de J aneiro, Rio de J aneiro, RJ .

UNITERMOS: Microsporidia. HIV. Características clínicas. Abordagem diagnóstica e terapêutica.

KEY WORDS: Microsporidia. HIV. Clinical features. Approach diagnostic and therapeutic.

\section{INTRODUÇÃO}

Desde o surgimento da síndrome de imunodeficiência adquirida (SIDA) em 1981, passamos a observar nesses pacientes infecções causadas por patógenos oportunistas, antes descritas apenas em pacientes com outras causas de imunodeficiência, como neoplasias, uso de drogas imunossupressoras e transplantes de órgãos. Entre as parasitoses oportunistas observadas nos indivíduos com SIDA, as protozooses são as predominantes, em particular a toxoplasmose, as coccidioses intestinais e a pneumocistose. Até há alguns anos, as microsporidioses eram consideradas como infecções muito raras em humanos, ocorrendo apenas em pessoas imunodeprimidas. A identificação em 1985 de uma nova espécie parasita do homem (Enterocytozoon bieneusi ) e, a seguir, a observação de casos de ceratoconjuntivite devido ao E ncephalitozoon em pacientes com SIDA relevaram o papel patogênico do microsporidium.

O espectro clínico das doenças causadas por microsporidia encontra-se em expansão. O gênero Enterocytozoon é o mais isolado em pacientes com diarréia crônica e SIDA. Recentemente, a espécie Septata intestinalis, também conhecida como “Encephalitozoon intestinalis"1, foi descrita em células do epitélio intestinal, células tubulares renais e respiratórias de pacientes HIV-positivos, indicando disseminação sistêmica.

No Brasil, de forma semelhante a outros locais no mundo, as infecções intestinais são as mais comuns, com 40 casos descritos ${ }^{2-4}$. No Rio de J aneiro e em São Paulo, dois casos de microsporidiose ocular já foram diagnosticados ${ }^{5,6}$. É possível que um número maior de casos seja identificado à medida que se conheça melhor a distribuição, ca-

\begin{tabular}{|lcc|}
\hline Tabela 1-Classificação dos microsporidia \\
\hline Phylum & \multicolumn{2}{c|}{ Microspora } \\
Classe & \multicolumn{2}{|c|}{ Microsporea } \\
Ordem & \multicolumn{2}{|c|}{ Microsporidia } \\
Subordem & Pansporoblastina & Apansporoblastina \\
Gêneros & Pleistophora & Nosema \\
& Septata & Enterocytozoon \\
& & Encephalitozoon \\
Espécies & S. intestinalis* & N. connori \\
& & N. corneum \\
& & E. bieneusi \\
& & E. cuniculi \\
& & E. hellem \\
\hline * Classificada por alguns autores em Encephalitozoon intestinalis ${ }^{*}$. \\
\hline
\end{tabular}

racterísticas clínicas e métodos diagnósticos da infecção por esse parasita. Neste artigo, procuramos abordar os aspectos gerais da microsporidiose humana, para que com maior conhecimento da infecção possamos estabelecer sua prevalência, e definir melhor sua participação na história natural do paciente co-infectado pelo HIV no Brasil.

\section{Microsporidia}

O Nosema bombycis foi o primeiro dos mi crosporidia descrito ${ }^{7}$, sendo agente parasita do bicho-daseda. A partir de então esses agentes têm sido reconhecidos como causa de doença em vários grupos de animais: insetos, peixes, coelhos, ursos e primatas ${ }^{8,9}$. De fato, os mi crosporidia estão amplamente distribuídos no reino animal, parasitando todas as classes de vertebrados e invertebrados ${ }^{10,11}$.

\section{Características biológi cas e ciclo de vida}

Os microsporidia são protozoários pertencentes ao phyl um Microspora (tabela 1), termo criado em 1882 por Balbini para a classificação de um novo grupo de parasitas ${ }^{12,13}$ : eucariotas primitivos, desprovidos de mitocôndrias, cujo desenvol vi mento se faz por divisão múltipla ou merogonia, seguida de esporogonia, no interior da célula do hospedeiro. Essa divisão parasitária efetua-se, na maioria dos gêneros, em contato direto com o citoplasma da célula do hospedeiro (subordem Apansporoblas- 
tina: gêneros Nosema, Enterocytozoon e Encephalitozoon). Alguns se multiplicam dentro de um vacúolo parasitóforo, cuja membrana é de origem celular parasitária (subordem Pansporoblastina: gêneros Pleistophora e Septata). A esporogonia leva à formação de esporos unicelulares maduros, de tamanho de 1 a 1,5 $\mu \mathrm{m}$, com um túbulo polar espiralado e cercado de uma membrana espessa. A contaminação de novas células se faz pela adesão desses esporos na membrana, seguida da expulsão, por mecanismo de ejeção, do túbulo polar, através do qual passa o esporoplasma, elemento infeccioso. E sse ti po de esporogonia é característico dos microsporidia, assim como o modo de contaminação. Mais de 100 gêneros e quase 1.000 espécies foram até agora identificados ${ }^{14}$.

A classifi cação das espécies é ai nda baseada em características ultra-estruturais, isto é, no tamanho e morfologia dos diferentes estágios de desenvolvimento, na configuração do núcleo e no número de espirais do túbulo polar. Outro critério também usado para a classificação é a especificidade do local de desenvolvimento: algumas espécies são restritas a uma célula específica de um único órgão ou sistema; outras causam infecção sistêmica, envolvendo difer entes órgãos e sistemas. Esses critéri os de classifi cação, entretanto, têm si do objeto de revisão, sobretudo após a introdução da análise molecular, cujos estudos, ainda incompletos, por vezes não coincidem com os estudos morfológi $\cos ^{1}$.

\section{EPIDE MIOLOGIA}

A epidemiologia da microsporidiose humana varia de acordo com o estado i mune do hospedeiro e com a espécie do parasita. As dificuldades diagnósticas, na dependência da demonstração morfológica do organismo, limitam a realização de inquéritos epidemiológicos. A interpretação e utilidade da soroprevalência são controversas. A alta prevalência de soropositividade ao Encephalitozoon cuniculi, sem correlação clínica ou epidemiológica, assim como a possibilidade de reações cruzadas entre diferentes antígenos de microsporidia, dificulta a sua interpretação ${ }^{15}$.

\section{Prevalência e distribuição geográfica}

Somente a partir de 1959 o microsporidium foi reconhecido como agente de patol ogia humana, com o relato de um caso ${ }^{16}$. A seguir, apenas dez casos, não associados ao HIV, foram descritos ${ }^{1,17-19}$. Com a progressão da epidemia da SIDA, mais de 600 casos foram relatados somente nos Estados U nidos ${ }^{17}$. Até agora cinco gêneros foram identificados como causas de doença no homem: Nosema, Pleistophora,
Encephalitozoon, Enterocytozoon e, mais recentemente, Septata ${ }^{20-26}$. O Enter ocytozoon bieneusi, previamente exclusivo de indivíduos infectados pelo HIV, foi recentemente detectado como causa de diarréia autolimitada em viajante imunocompetente ${ }^{19}$, e em paciente com imunossupressão secundária à quimioterapia pré-transplante ${ }^{1}$. Relatos de infecção por microsporidia desse gênero em indivíduos assintomáticos sugerem a possibilidade de existência de portadores sãos do parasita 27,28 .

No indivíduo infectado pel o HIV, 4 dos 5 gêneros foram identificados como causa de microsporidiose: Pleistophora, Encephalitozoon (E. cuniculi, E. hellem), Enterocytozoon ( $E$. bi eneusi) e Septata (S. intestinalis), sendo a maioria causada pelo Enterocytozoon bieneusi, associada a sintomas digestivos. A prevalência da infecção por microsporidia nos pacientes com SIDA e diarréia crônica varia de $7 \%$ a $50 \%$ no mundo, com ampla distribuição geográfica: Alemanha, Austrália, Brasil, Canadá, Estados Unidos, França, Inglaterra, Itália, Países Baixos, Porto Rico, Suíça, Zâmbia 2,17,19,21,29-38. Essas diferenças de prevalência podem refletir diversidade de exposi ção, emprego de diferentes técnicas diagnósticas ou variação geográfica. A propósito da alta incidência dessa infecção, alguns autores acreditam que o $E$. bieneusi seja um parasita natural do homem, infectando transitoriamente o indivíduo com o sistema imune competente, causando doença somente em imunodeprimidos ${ }^{18}$.

\section{Fontes de infecção e transmissão}

As fontes de infecção e os modos de transmissão da microsporidiose são incertos. Não há confirmação da presença de um reservatório animal na infecção humana, nem de hospedei ros intermediários ou vetores invertebrados ${ }^{8,10,18}$. A presença de microsporidia na superfície de águas poderia sugerir a possibilidade do meio ambiente como fonte de infecção, não fosse a ausência de identificação de espécies que infectam o homem nesses locais ${ }^{39}$.

A identificação de esporos do parasita na urina, fezes, aspirados duodenais e secreções respiratórias sugere a possibilidade de transmissão interhumana. A ingestão de esporos seria uma explicação plausível para a aquisição de espécies como E. bieneusi e $\mathrm{S}$. intestinalis, já que esses esporos se desenvol vem principal mente no intestino del gado e são excretados nas fezes. Além disso, a identificação desses dois gêneros somente em humanos confirmaria a hipótese de transmissão de pessoa para pessoa. A disseminação de S. intestinalis, E. hellem e E. cuniculi para o sistema urinário, além de infecção prostática, levantaria a possibilidade adicional da transmissão sexual ${ }^{37}$. E specula-se a possi- 
bilidade de transmissão aérea pela presença de esporos de $\mathrm{E}$. bieneusi, $\mathrm{E}$. hellem e $\mathrm{E}$. cuniculi nas vias respiratórias ${ }^{37,40,41}$. A infecção da conjuntiva ocular por Encephalitozoon faz da auto-inoculação sua hi pótese de transmissão mais provável ${ }^{17}$. Apesar da infecção por via transplacentária ser demonstrada em animais de experimentação, a transmissão vertical nunca foi demonstrada em humanos ${ }^{1,42}$.

\section{PATOGE NICIDADE}

A fisiopatologia da microsporidiose humana não é completamente entendida. Categorias diversas de interação parasita-hospedeiro foram descritas entre humanos e o Encephalytozoon, variando de infecção latente assintomática ou infecção crônica moderada à doença aguda potencialmente fatal, em função do estado imune do hospedeiro ${ }^{25}$. O papel de uma resposta celular efetiva em reduzir a multiplicação dos microsporidia já foi estabelecido experimentalmente. Análises epidemiológicas sugerem que pacientes com imunodeficiência celular grave apresentam um risco maior de desenvolver doença por microsporidia, principal mente quando associada à redução de células do tipo $\mathrm{CD} 4^{1}$.

Enquanto o Encephalitozoon se prestaria a estudos comparativos de infecção entre animais e humanos, não fossem dificuldades básicas como espécies do parasita e quali dade de resposta imune do hospedeiro diferentes, a ausência de modelo animal para o estudo do $\mathrm{E}$. bi eneusi faz com que a compreensão da sua patogênese dependa exclusivamente de estudos clínicos descritivos e análises epidemiológicas da infecção. O progresso no entendimento do papel do microsporidium na infecção humana esbarra sobretudo no problema diagnóstico, na dependência da existência de um método simples, não invasivo, sensível, específico e quantitativo, a fim de que se possa comparar a preval ência e intensidade da infecção entre grupos sintomáticos e assintomáticos. Estudos iniciais envolvendo pacientes com SIDA mostraram uma correlação entre diarréia crônica sem etiolo-

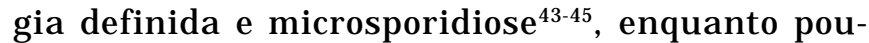
cos estudos posteriores não encontraram essa associação ${ }^{27}$. A presença de co-infecção intestinal encontrada em até um terço das microsporidioses intestinais, além da existência de portadores assintomáticos de microsporidia, col oca em questão o papel patogênico desse parasita ${ }^{1}$. A prevalência de microsporidiose em pacientes infectados pelo HIV ainda deve ser adequadamente estudada em função de todo espectro clínico e imunológico, naturais da evolução da síndrome.

Tabela 2 - Gênero e espécie de microsporidia e manifestações clínicas na infecção humana

Gênero e espécie de microsporidia

Enterocytozoon bieneusi

Septata intestinalis*

Encephalitozoon cuniculi

Encephalitozoon hellem

Pleitosphora sp.

Nosema connori

Nosema corneum Microsporidium ceylonensis Microsporidium africanum

* Classificada por alguns autores em Encephalitozoon intestinalis ${ }^{1}$

\section{MANIFESTAÇÕES CLÍNICAS}

As manifestações clínicas da microsporidiose humana variam desde o acometimento ocular, muscular ou intestinal isolados, até o comprometimento sistêmico (tabela 2). No indivíduo infectado pelo HIV, a manifestação mais prevalente é a intestinal, com síndrome diarréica. No entanto, a disseminação dos parasitas dos gêneros Encephalitozoon e Septata vem sendo cada vez mais identificada ${ }^{37,41,46}$.

\section{Microsporidiose não relacionada à SIDA}

F oram até hoje relatados três padrões de infecção por microsporidia, de acordo com o estado imune do hospedeiro:

\section{MICROSPORIDIOSE SISTÊMICA OU MUSCULAR, NA IMU- NODEFICIÊNCIA CELULAR}

Esse padrão de infecção foi apresentado por duas crianças, de 9 e 2 anos de idade, em 1959, no J apão, e em 1984, na Suécia, respectivamente, com acometimento do SNC e apresentação clínica predominante de crises convulsivas. Foi identificada a presença de Encephalitozoon spp. no LCR e na urina da primeira criança e apenas na urina da segunda criança. Apesar da presença constante do parasita na urina, elas não apresentavam sintomas urinários ou alterações das provas de função renal ${ }^{16,47}$. Outro caso de possível disseminação do parasita foi relatado, em 1973, em autópsia de um lactente de 4 meses de idade com aplasia tímica que apresentava, além de diarréia crônica, febre, perda de peso e dispnéia. Nosema connori foi identificado nos pulmões do lactente, que apresentava 
também Pneumocystis carinii ${ }^{24}$.

Miosite por Pleistophora sp., descrita em 1985 em paciente com imunodeficiência celular grave, embora soronegativo para HIV: jovem de 20 anos de idade com perda muscular progressiva, febre, adenopatia generalizada e perda de peso; não apresentava dor. Quatro anos mais tarde foi confirmada a ausência de reatividade ao HIV, mas o paciente mantinha deficiência de imunidade celular de causa desconhecida. Miopatia difusa foi confirmada por estudos de condução nervosa e el etromiografia ${ }^{48}$.

\section{INFECÇÃo da CÓRNEA EM IMUNOCOMPETENTES}

A presença de Nosema corneum e N osema ocularum ou microsporidia do tipo Nosema (M. africanum e $M$. ceylonensis) no estroma da córnea de quatro indivíduos com queixas de redução da acuidade visual, borramento e dor caracteriza as ceratites ou ceratoconjuntivites por microsporidia nesses pacientes. Somente um deles tinha história de trauma prévio e presença de corpo estranho, retirado no momento da biópsia. Não houve resposta à terapia tópica, mas somente à ceratoplastia e ao transplante de córnea realizado em dois pacientes ${ }^{49,50}$.

\section{Microsporidiose como infecção oportunista na SIDA}

As manifestações clínicas variam de assintomáticas ou moderadas às doenças debilitantes, envolvendo os ol hos e o trato intestinal, respiratório e urogenital deste grupo de indivíduos. As infecções intestinais e biliares devido ao E. bieneusi são as mais freqüentes, seguidas pelas manifestações sistêmi cas por E. hellem, E. cuni culi e Septata intestinalis.

O espectro de doenças causadas pel o Encephal itozoon spp. tem-se expandido, incluindo: ceratoconjuntivite, sinusite, bronquiolite, nefrite, uretrite, cistite, hepatite e peritonite ${ }^{1,15,36,37,41,51-53}$. Um único caso de miosite por Pleistophora sp. em paciente HIV positivo foi descrito em $1993^{53}$.

\section{MICROSPORIDIOSE DO TRATO BILIAR E INTESTINAL}

$A$ infecção por $E$. bieneusi ainda não pode ser definida como causa de diarréia entre os pacientes HIV positivos, embora existam relatos da associação microsporidiose e diarréia crônica, também chamada de persistente (três ou mais evacuações/ dia, de consi stência alterada, por um período mínimo de um mês ${ }^{55}$ ), sem sangue, muco ou pus; sem febre; acompanhada de anorexia, perda de peso de cerca de $2 \mathrm{~kg} / \mathrm{mês}$. Alguns pacientes apresentam diarréia de caráter intermitente e poucos eliminam os esporos na ausência de diarréia ${ }^{27,56-59}$. Po- dem ocorrer náuseas, vômitos e dor abdominal. A duração média dos sintomas vai de 1 a 31 meses $^{56}$. Os achados de exame físico parecem inespecíficos. O acompanhamento a longo prazo, de até três anos, de alguns pacientes infectados pelo $\mathrm{E}$. bieneusi mostra que a infecção pode ter um curso benigno ${ }^{28,60}$, ou ainda ser um co-fator de consumo na caquexia da fase terminal de alguns pacientes $^{58,61}$. Até $1 / 3$ dos pacientes com infecção por microsporidia apresenta co-infecção intestinal ${ }^{1}$.

A disseminação extra-intestinal do $\mathrm{E}$. bieneusi clinicamente sintomática é incomum, mas o parasita já foi detectado na árvore biliar de indivíduos com colangite e colecistite acalculosa62-64. Como o citomegal ovírus e o Cryptosporidium, os mi crosporidia podem ser responsáveis pela colangiopatia associada à SIDA. Na maioria desses casos, a diarréia também está presente e não há sinais clínicos de acometimento hepático ${ }^{1}$. UItra-sonografia ou tomografia computadorizada de abdome revela dilatação das vias biliares, espessamento de parede da vesícula e até lama biliar. Os níveis séricos de fosfatase alcalina podem estar elevados com transaminases e bilirrubinas normais ${ }^{64}$. Os níveis séricos de albumina não estão constantemente reduzidos, apesar da longa duração da diarréi $a^{56}$.

A infecção por $\mathrm{E}$. bi eneusi, geralmente, acontece quando os níveis de CD4 circulantes caem abaixo de 50 a 100 células $/ \mathrm{mm}^{3}$. Quando a microsporidiose é identificada, a maioria dos pacientes já apresentou outra infecção oportunista ${ }^{1}$. A infecção por microsporidia parece responsável pela definição da síndrome em cerca de $25 \%$ dos $\operatorname{casos}^{56}$.

S. intestinalis também causa diarréia crônica com perda de peso e disseminação para vias biliares de maneira semelhante ao $E$. bieneusi. No entanto, ainda que leve à disseminação sistêmica para rins e outros locais, sem conexão com o intestino ${ }^{1}$, a queixa primária na infecção por essa espécie de microsporidium também é a diarréia ${ }^{33}$.

\section{Hepatite e peritonite}

Em 1987, Terada descreveu o primeiro caso de hepatite por E. cuniculi em um paciente de 35 anos infectado pelo HIV, com sarcoma de Kaposi e contagem de $C D 4=48$ células $/ \mathrm{mm}^{3}$. O indivíduo apresentava, inicialmente, síndrome diarréica intermitente, seguida, quatro meses mais tarde, de hepatite fulminante e diarréia grave, levando ao óbito ${ }^{52}$.

A peritonite por E. cuniculi foi descrita em 1989 por Zender em um paciente de 45 anos, com contagem de CD4 igual a 57 células $/ \mathrm{mm}^{3}$. Internado com pneumocistose, evoluiu com febre persistente e aparecimento de massa palpável no abdome. Desenvolveu quadro de íleo paralítico, com óbito 40 
dias após a admissão. A autópsia mostrou uma massa não granulomatosa em peritônio, contendo microsporidia cuja ultra-estrutura foi compatível com a do E. cuniculi ${ }^{53}$.

\section{Microsporidiose RESPIRATÓRIA}

A maioria das microspori dioses do trato respiratório faz parte de uma síndrome intestinal ou sistêmica. Sinusites, com ou sem pólipos nasais, são referidas como manifestações predominantes de casos de disseminação por E. bieneusi, Encephalitozoon spp. ou S. intestinali ${ }^{65,67}$ e apresentam-se como obstrução e descarga nasal mucopurulenta. Esses microsporidia foram também identificados no trato respiratório inferior, raramente causando bronquiolite, pneumonia ou insuficiência respiratória aguda. Existe o relato de um caso de pneumonia por $\mathrm{E}$. bieneusi em paciente com diarréia crônica que desenvolveu tosse persistente, com expectoração clara, dispnéia e sibilos. Sua radiografia de tórax mostrava um infiltrado intersticial discreto, associado a pequeno derrame pleural. Foram detectados esporos de $\mathrm{E}$. bieneusi no lavado bronco-alveolar (LBA), em biópsia transbrônquica do segmento posterior do lobo pulmonar inferior esquerdo, em fragmento de biópsia realizada em íleo e nas fezes ${ }^{40}$. Outros dois casos de pneumonia intersticial, sintomática, por Encephalitozoon foram documentados como parte de comprometimento sistêmico ${ }^{46,51}$. A col onização do trato respiratóri o foi demonstrada pel a observação de um paciente assintomático com esporos de $\mathrm{E}$. hel lem no escar$\mathrm{ro}^{37}$. Não é conhecida a prevalência de colonização pulmonar por microsporidia, mas não é freqüente a manifestação pulmonar ${ }^{1}$. I lustração disso é a importância da infecção por $\mathrm{S}$. intestinalis na doença respiratória, já detectada em material de autópsia de células brônquicas epiteliais ${ }^{34,68}$.

\section{MICROSPORIDIOSE URINÁRIA}

Os efeitos da presença de $\mathrm{E}$. hellem ou S. intestinalis nos sistemas renal e urinário levam a manifestações clínicas pouco conhecidas e se restringem a observações prel iminares de que podem ser infecções latentes, com ou sem micro-hematúria assintomática ${ }^{37}$. Cistite, nefrite intersticial com disúria e hematúria maciça também já foram descritas $^{41}$. Microsporidia já foram observados na próstata de um paciente com encefalitozoonose sistêmica ${ }^{69}$. Também a microsporidiose urinária parece fazer parte de uma disseminação sistêmica do parasita.

\section{MICROSPORIDIOSE SISTÊ MICA}

A fonte de infecção e o modo de transmissão da microsporidiose humana não são conhecidos. Em model os experimentais com o $\mathrm{E}$. cuniculi, foi demonstrado que o sítio primário da infecção é o enter ócito do intestino del gado, a partir do qual os parasitas atingem células endoteliais e macrófagos e disseminam-se para todos os órgãos, atingindo até mesmo os pulmões, com uma predileção para rins e cérebro ${ }^{8}$. Tais modelos explicariam o comprometimento multissistêmico dos relatos da infecção por E . hellem, E . cuni culi e S. intestinalis nos indivíduos HIV+, com contagem de CD4 inferior a 50 cél ulas/ $/ \mathrm{mm}^{3} \quad 34,37,41,46,56,69$.

\section{Encephalitozoon spp.}

As manifestações clínicas da infecção por Encephal itozoon variam de assintomáticas a sistêmicas.

As duas espécies de Encephalitozoon - E. hellem e E. cuniculi - só recentemente foram diferenciadas com base em estudos antigênicos e análises bioquímicas, uma vez que são morfologicamente semelhantes. O modelo característico de infecção sistêmica por E. hellem consiste de ceratoconjuntivite e manifestações do trato respiratório e urinário. Acometimento orgâni co múlti plo foi achado de autópsia em indivíduo hispânico com SIDA, de 30 anos de idade, em 1992. O paciente apresentou quadro de dor abdominal, disúria e hematúria maciça, evoluindo com insuficiência renal e respiratória progressivas ${ }^{41}$. Esporos de E. hellem foram encontrados na córnea, trato urinário, e trato respiratório na autópsia do paciente. Grande quantidade de parasi ta foi vista no epitél io de quase toda a extensão da árvore respiratória. A espécie foi identificada por meio da reação com anti-soro específico na cultura dos esporos obtidos a partir da urina do paciente, antes do óbito. A confirmação da espécie se deu mediante ensaio de imunofluorescência indireta em corte de tecido parafinizado dos rins e pulmões ${ }^{41}$. Se a presença de microsporidia disseminados contribui para o agravamento ou progressão fatal da doença não é sabido, já que em culturas de tecido pós-morte cresceram também bactérias patogênicas, como Klebsiella pneumoniae e $E$. coli, além de terem sido identificados corpos de inclusão citomegálica e micobacteriose disseminados ${ }^{41}$.

\section{Ceratoconj untivite na Sida}

A infecção ocular por microsporidia nos indivíduos infectados pelo HIV é limitada ao epitélio superficial da córnea e conjuntiva ${ }^{36}$. E. hellem é a espécie mais freqüentemente identificada ${ }^{36}$. A apresentação clínica caracteriza-se por ceratite epitelial bilateral, punctiforme, além de hiperemia conjuntival associada à sensação de corpo 


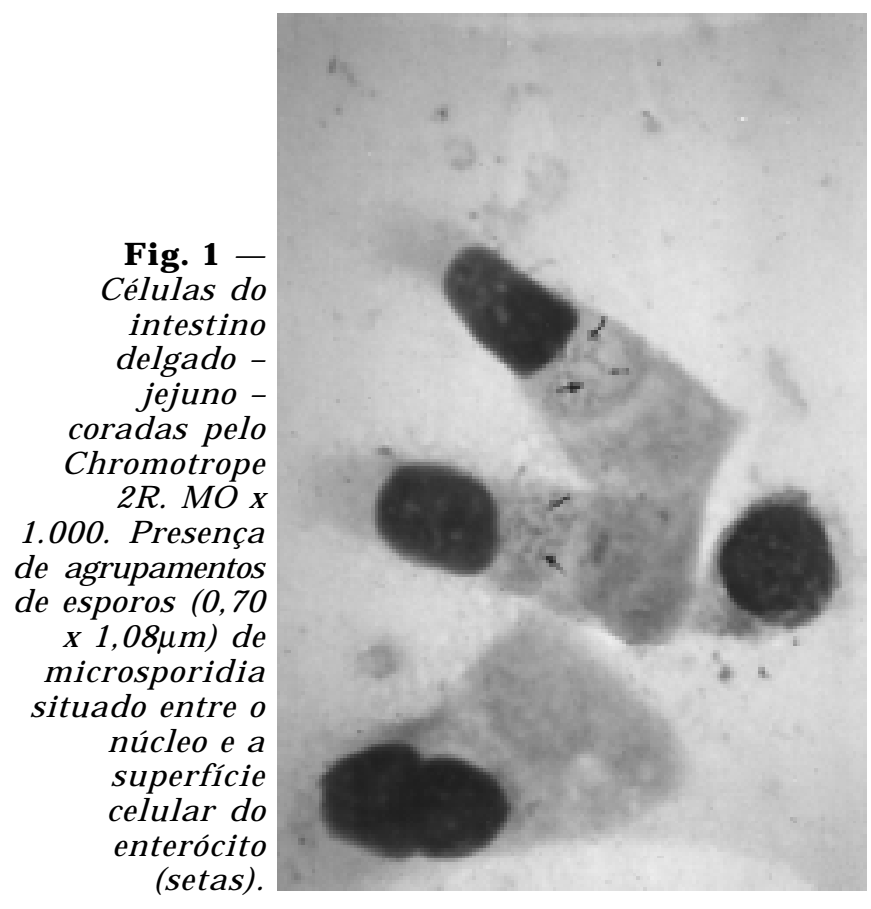

estranho, fotofobia e redução da acuidade visual. Pode ter caráter intermitente, com sintomatol ogia discreta, e raramente leva à ulceração da córnea ${ }^{1}$. Dos sete casos até hoje relatados, todos apresentavam microsporidia também na urina ou trato respiratório, sugerindo quadro de disseminação sistêmi ca ${ }^{37,41,51,66,70,71}$.

\section{S. intestinalis (E. intestinalis)}

I nicialmente confinada ao intestino delgado e trato biliar, pode, também, atingir rins e trato respiratório. Não parece, porém, ter muita representação clínica na infecção sistêmica, exceto por um relato de nefrite intersticial ${ }^{34}$, outro de urgência urinária associada a piúria estéril e elevação da creatinina sérica ${ }^{72}$, além de casos crescentes de sinusite e conjuntivite ${ }^{33}$.

Nos relatos de microsporidiose humana na SIDA, observa-se que a infecção pode ter um comportamento ambíguo em relação às manifestações clínicas, que variam de benignas, com curso arrastado, às apresentações fulminantes, quando não fica claro o papel patogênico do microsporidium. A presença de portadores assintomáticos sugere que o parasi ti smo seja do tipo oportunista, com quadro clínico só compl etamente caracterizado na presença de imunodepressão celular acentuada.

\section{DIAGNÓSTICO}

O diagnóstico da infecção por microsporidia depende da demonstração morfológica do parasita por microscopia óptica (MO) ou eletrônica (ME).

Exame direto - A microscopia óptica pode ser suficiente na maioria dos $\operatorname{casos}^{73}$. O achado de esporos do parasita em amostras col hi das no local da infecção ou em líquidos corporais: swab conjuntival, secreção respiratória e de seios da face, LBA, urina, aspirado duodenal ou fezes, embora sensíveis com a utilização de col orações especiais, dependem de examinador especializado. Os esporos de tamanho muito pequeno $(0,2-1,2 \times 1,5 \mu \mathrm{m})$ são identificados no exame direto através de leitura em microscopia óptica, aumento $=1.000 \mathrm{x}$, corados com chromotrope ${ }^{74}$; gram-chromotrope (Moura et al. 1996 - em publicação) ou substâncias quimioluminescentes ${ }^{71}$, que permitem a distinção entre os esporos e as bactérias ou leveduras. A coloração pelo Giemsa ${ }^{77}$ pode ser utilizada na visualização do organismo em urina, swab conjuntival e LBA, graças ao tamanho maior do esporo $(2,5-3,0 \times 1,3-2,0 \mu \mathrm{m})$ das espécies que acometem os respectivos órgãos, diferente dos encontrados nas fezes, muito menores.

Biópsias - Tecidos adquiridos por biópsias são, normalmente, processados em parafina com cortes finos ou semifinos e corados com Giemsa, Hematoxilina-eosina, Warthin-Starry, Brenner ou PAS. No intestino, o parasita é encontrado na região supranuclear da célula epitelial (fig. 1) ou dentro dos macrófagos da lâmina própria.

A identificação da espécie é feita mediante exame de cortes ultrafinos do tecido em microscopia el etrônica, exame bastante oneroso e menos sensível que o método direto, mas que permite a classificação ultra-estrutural do parasita, com identificação das características dos seus estágios de desenvolvimento (fig. 2). A menor sensibilidade da microscopia el etrônica de transmissão (TEM) devese ao fato de que é ger al mente feita com fragmento de biópsia selecionado pelo endoscopista, mas que pode não representar, enfim, o sítio infectado. A TEM pode, também, ser utilizada em amostras provenientes de líquidos corporais, assim como fezes e urina, para a diferenciação dos esporos de $\mathrm{E}$. bieneusi e $\mathrm{S}$. intestinalis, mas não dos esporos de Encephalitozoon e S. intestinalis ultra-estruturalmente muito semel hantes.

Sorol ogia: só pode ser feita com as formas cultiváveis de Encephalitozoon ou Septata, mas está prejudicada nesses pacientes, devido à imunodeficiência, que torna irregular a produção de anticorpos.

Imunofluorescência - Anticorpos policlonais marcados com fluoresceína têm sido usados por alguns pesquisadores para detecção histológica e citológica de microsporidia das espécies humanas e para a visualização de diferentes estágios em 

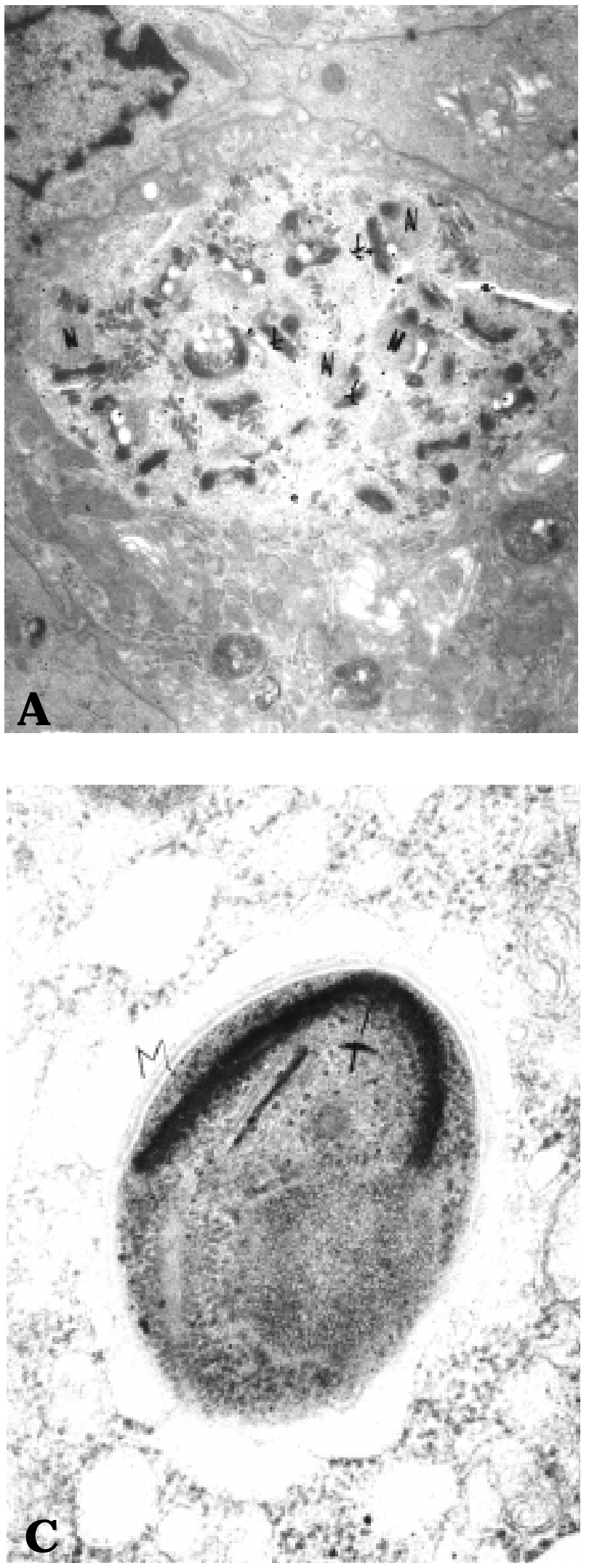

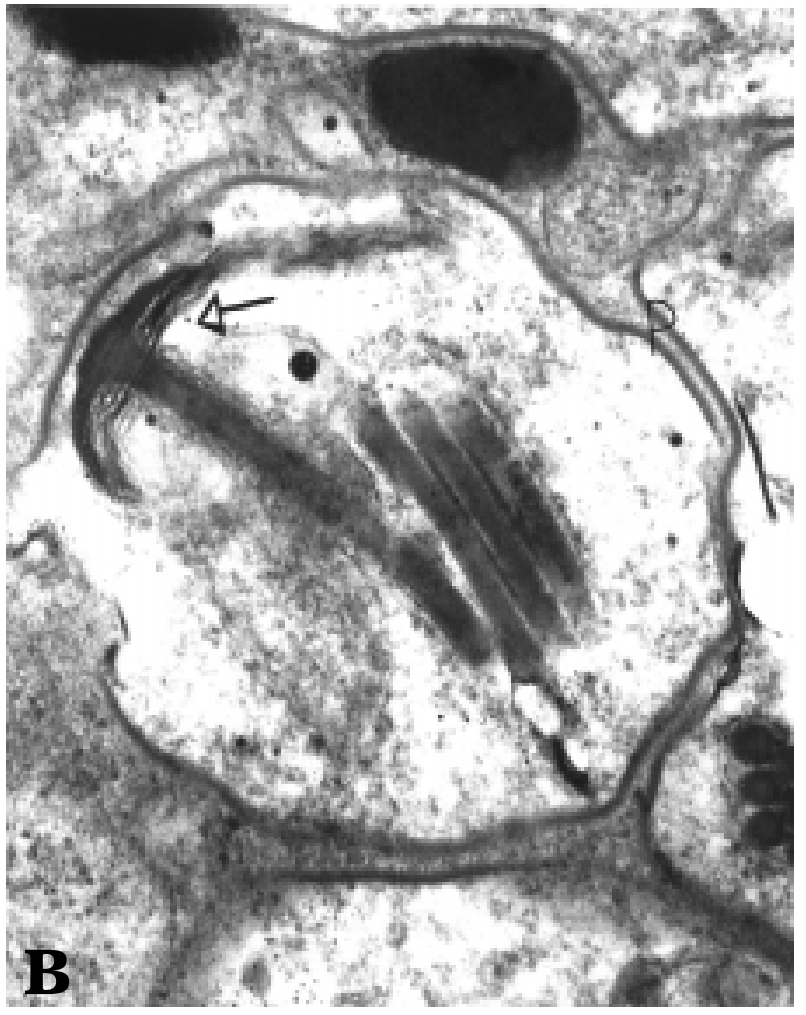

Fig. 2 - Estágios de maturação do microsporidium no interior do enterócito (TEM):

A) esporogonia: reconhecida pela presença de estruturas

el etronicamente densas, precursores dos túbulos polares (t). Núcleos (N). Vacúol os el étron-luscentes (e). x15.000; B) à medida que o espor onte amadurece, individualiza-se através da invaginação do plasmalema $(P)$. Cada núcleo se torna isolado acompanhado de um túbulo polar denso, em forma de âncora (seta) x140.000;

C) Esporo, com seu túbulo polar (T) e membrana espessa (M). x100.000. 
cultura de células. A reatividade cruzada entre os antígenos do Encephalitozoon e do E. bieneusi permite a visualização de anticorpos para a demonstração de esporos de $\mathrm{E}$. bi eneusi nas fezes e em tecidos ${ }^{76}$.

Biologia molecular - O diagnóstico molecular só é possível em laboratórios de pesquisa que dispõem de primers de alguns microsporidia humanos estudados.

\section{$\mathrm{Na}$ suspeita de microsporidiose intestinal}

A introdução de métodos de fácil realização, menor custo e maior conforto para o paciente muda a perspectiva de abordagem diagnóstica do indivíduo HIV + com diarréia crônica. Outrora, a limitação da investigação parasitológica fazia da biópsia intestinal indicação para os casos com rotina repetidamente negativa. A pesquisa de microsporidia nas fezes contribuiu para a ampliação do espectro de agentes etiológi cos da diarréia em SIDA. Dados epidemiológi cos sugerem que as técnicas disponíveis para o exame direto são mais sensíveis que o exame de fragmentos de biópsias ${ }^{28,74,77}$. No entanto, não se sabe se a excreção de esporos de microsporidia é intermitente; um número maior de amostras analisadas poderia resultar no aumento da sensibilidade do método. Além disso, a quantidade mínima de esporos que pode ser detectada no exame direto ainda não foi determinada. Por isso, o momento e a necessidade de realização de biópsias ou obtenção de aspirados ainda são discutidos. Apesar de sempre referidos no jejuno ou duodeno, esporos do parasita são encontrados também no íleo ${ }^{60,78}$ e, raramente, no cólon ${ }^{27}$. A colonoscopia, porém, permanece como um importante método de diagnóstico da diarréia crônica, pois detecta infecções tratáveis e não diagnosticadas pelo exame de fezes, como a citomegalovirose intestinal ${ }^{79}$, podendo ser estendida ao íleo, o que permite o diagnóstico, também, da microsporidiose intestinal, poupando o paciente de futura endoscopia al ta $^{74}$.

\section{Na suspeita de microsporidiose ocular}

$\mathrm{Na}$ maioria dos casos de conjuntivite por Encephalitozoon, um exame direto de swab da conjuntiva ocular com col oração pelo chromotrope, Gram ou Giemsa é suficiente para o diagnóstico ${ }^{36,37}$. Microsporidia já foi isolada de amostras de conjuntiva humana também com a utilização de cultura de células ${ }^{26}$. $\mathrm{Na}$ confirmação de microsporidiose ocular, deve ser feita pesquisa de microsporidium na urina e LBA, já que pode fazer parte de quadro de disseminação do parasita.

\section{TRATAME NTO}

A avaliação de drogas in vitro e o estudo do tratamento da microsporidiose em animais são limitados $8,14,50$. A fumagilina reduz a infecção por mi crospori dium da abel ha ${ }^{80}$ e inibe a replicação do Encephalitozoon spp. em cultura de células in vitro $^{18}$ e na infecção experimental de coel hos ${ }^{81}$, apesar de nunca erradicar o parasita. A fumagilina tem sido estudada como droga humana antiprotozoário, mas ainda não foi licenciada para uso humano ${ }^{1}$.

A experiência clínica no tratamento da microsporidiose humana é reduzida e faltam ensai os duplo-cegos com a utilização de placebos para avaliação de uma droga anti parasitária eficaz. No entanto, existem diversas observações sobre o sucesso terapêutico do albendazol na infecção por Septa$\operatorname{ta}^{1,30,33,56,82}$.

Os benzimidazólicos, ou albendazol, ligam-se com a tubulina, inibindo sua polimerização em microtúbulos, interferindo com a aquisição de nutrientes e a divisão celular. A divisão do mi crosporidium acontece na fase de merogonia e esporogonia, e parece que o al bendazol levaria à degeneração desses estágios proliferativos. Esporoblastos e alguns esporos maduros são vistos após o tratamento com essa droga, que parece ter efeito mais parasitostático do que parasiticida. A inibição da reprodução do mi crosporidium seria incompleta com as doses geral mente utilizadas, em função da reduzida penetração celular dos medicamentos ${ }^{29}$.

A experiência no tratamento da infecção por Septata intestinalis é ainda pequena, com um relato de pouco mais de 30 casos. Um curso de 2 a 4 semanas de al bendazol, $400 \mathrm{mg} /$ dose, duas vezes ao dia, leva à mel hora clínica, em paralel o com o desaparecimento dos esporos de Septata intestinalis na urina e nas fezes dos pacientes infectados. Foi documentado, também, o desaparecimento do parasita do tecido intestinal ${ }^{30}$. É desconhecida a necessidade de esquema terapêutico de manutenção.

Ao contrário da $\mathrm{S}$. intestinalis, a experiência adquirida com o tratamento da infecção pelo $\mathrm{E}$. bieneusi mostra que ele tem efeito parcial. Estudos preliminares mostraram uma boa resposta clínica com o uso de metronidazol ${ }^{61}$, mas que não foram confirmados ${ }^{1}$.

Azitromicina ${ }^{30}$, atovaquona ${ }^{82}$ e outros antibióticos ou antiprotozoários têm sido usados, sem sucesso ${ }^{82}$. Relatos recentes têm mostrado que o tratamento da microsporidiose por $\mathrm{E}$. bieneusi com albendazol, 400mg, duas vezes ao dia, por período igual ou superior a quatro semanas, pode levar a significante mel hora clínica em alguns pacientes, ainda 
que não leve à eliminação do parasita no tecido intestinal, com continuação da eliminação de esporos nas fezes após o tratamento $29,56,68,82$.

Octreotídeo, droga usada no tratamento paliativo de diarréia crônica associada ao IIV $^{83}$, também se mostrou útil no tratamento de diarréia por microsporidia refratária ao tratamento com outras drogas ${ }^{84}$. Excreção esporádica de esporos de $E$. bieneusi, com melhora intermitente da diarréia, também foi observada sem nenhuma intervenção terapêutica ${ }^{1}$. No entanto, o desaparecimento espontâneo ou erradicação do parasita por longos períodos de tempo após o tratamento nunca foi demonstrado ${ }^{1}$.

Os raros casos até hoje descritos de infecção disseminada por Encephalitozoon ${ }^{75}$ responderam bem ao tratamento com al bendazol ${ }^{38,46}$.

A aplicação tópica de fumagilina ( $F$ umidil B) na ceratoconjuntivite pelo $\mathrm{E}$. hellem está associada à melhora clínica, com redução dos achados clíni$\cos ^{70}$. Porém, há recorrência dos sintomas após a interrupção do tratamento ${ }^{70}$. Já foi observada, também, melhora clínica com o uso de itraconazol ${ }^{3}$, que não foi confirmada em estudo posterior ${ }^{70}$. Estudos controlados devem ser realizados, pois existe a possibilidade de a encefalitozoonose ocular ter curso benigno na ausência de qualquer tratamento ${ }^{1}$.

Parece que a taxa de recaída da infecção está relacionada ao estado imune do indivíduo. É mais comum ocorrer em relação à presença de esporos no sítio infectado do que haver recorrência clínica dos sintomas. A terapia de manutenção, até hoje, não mostrou resultados significantes ${ }^{33}$.

\section{COMENTÁRIOS FINAIS}

No Brasil foram relatados, até hoje, 48 casos de microsporidiose, sendo a grande maioria de microsporidiose intestinal: 19 no Rio de J aneiro, 5 em São Paulo e 16 no Ceará; todos em pacientes infectados pelo HIV ${ }^{2,4,85}$. Os dois únicos casos (São Paulo) em indivíduos com sorologia negativa para HIV eram ambos transplantados de medula óssea 5 . Dois casos de microsporidiose ocular (Rio de J aneiro e São Paulo) ${ }^{6,86}$ e três de microsporidiose urinária (Rio de J aneiro) foram também descritos $^{2}$. Um caso de possível disseminação sistêmica foi achado de autópsia com presença do parasita em vias urinárias e sistema nervoso central (comunicação pessoal).

0 registro crescente de relatos de microsporidiose se deve ao maior conhecimento acerca do diagnóstico da infecção. À medida em que se for aperfeiçoando e for reconhecida a eficácia do exame direto, poderá ser, finalmente, dispensada a demonstração do parasita em tecido de biópsia, metodologia invasiva e sofisticada. A partir de então, poderemos montar inquéritos epidemiológicos, com estudos controlados, para maior compreensão do papel patogênico do microsporidium junto à enteropatia associada ao HIV e participar dos ensaios terapêuticos, contribuindo para meIhor qualidade de vida do paciente com SIDA em fase avançada de imunodepressão.

\section{AGRADE CIMENTOS}

Os autores agradecem a Claudine Sarfati, que gentilmente nos ofereceu as fotografias aqui utilizadas (Professora da cadeira de Parasitologia da Universidade Paris VII e Médica do Serviço de Parasitologia e Micologia do Hôpital Saint-Louis, 1, Av. Claude Vellefaux 7510, Paris).

\section{REFERÊNCIAS BIBLIOGRÁFICAS}

1. Weber R, Bryan RT, Schwartz DA et al. Human microsporidial infections. Clin Microbiol Rev 1994; 7: 426-61.

2. Moura H, Hirschfeld MPM, Brasil $P$ et al. Microsporidiosis among AIDS patients in Brazil: preliminary results. Workshop on Microsporidiosis and Cryptosporidiosis in Immunodeficient Patients, 1993; 40: 251.

3. Yee RW, Tio FO, Martinez J A et al. Resolution of microsporidial epithelial keratopathy in a patient with AIDS. Ophthalmol 1991; 98: 196-201.

4. Hirschfeld MPM, Cury AE, Moura $H$ et al. Identificação de microsporidia em 5 pacientes com AIDS no Município de São Paulo. Anais do XIII Congresso Brasileiro de Parasitologia (Resumos), 1993; 2: 95.

5. Hirschfeld MPM, Moura H, Pinto PLS et al. Investigação de microsporidia em pacientes submetidos a transplante de medula óssea. XXX Congresso da Sociedade Brasileira de Medicina Tropical, 1994; 27(supl.1): 343.

6. Hirschfeld MPM, Miyaki C, Takata CS et al. Ceratite associada a Microspori dium e adenovírus em paciente com AIDS. XXXI Congresso da Sociedade Brasileira de Medicina Tropical, 1995; 37(supl.1): 245.

7. Naegeli KW. Üeber die neue Krankheit der Seidenraupe und verwandte Organismen. Bot Z 1857; 15: 760-1.

8. Canning EU, Lom J , Dykova I. The microsporidia of vertebrates. New York, Academic Press, 1986.

9. Shadduck J A, Pakes SP. Encephalitozoonosis (nosemantosis) and toxoplasmosis. Am J Pathol 1971; 64: 657-71.

10. Canning EU, Hollister WS. Microsporidia of mammals widespread pathogens or opportunistic curiosities? Parasitol Today 1987; 3: 262-73.

11. Shadduck J A. Human microsporidiosis and AIDS. Rev I nfect Dis 1989; 11: 203-7.

12. Balbiani M. Sur les microsporidies ou sporospermies des articules. CR Acad Sci 1882; 95: 1.168-71.

13. Kudo R. A biologic and taxonomic study of the Microsporidia. III Biol Monogr 1924; 9: 1-268.

14. Canning EU. Microsporidia. In Kreier J P, Baker J R (eds): Parasitic protozoa, $2^{\text {nd }}$ ed. New Y ork, Academic Press, 1993; 299-385.

15. Bryan RT. Microsporidia. In Mandell GL, Bennett J E, Dolin R (eds): Principles and practice of infectious diseases, $4^{\text {th }}$ ed. 
New York, Churchill Livingston, 1995; 1-26.

16. Matsubayashi $\mathrm{H}$, Koike $\mathrm{T}$, Mikata I et al. A case of Ence phalitozoon-like body in man. Arch Pathol 1959; 67: 181-7.

17. Bryan RT. Microsporidiosis as an AIDS-related opportunistic infection. Clin Infect Dis 1995; 21(suppl 1): S62-65.

18. Canning EU, Hollister WS. Human infections with Microsporidia. Rev Med Microbiol 1992; 3: 35-42.

19. Sandfort J, Hannemann A, Gelderblom H et al. Enterocytozoon bieneusi in an immunocompetent patient who had acute diarrhea and who was not infected with the human immunodeficiency virus. Clin I nfect Dis 1994; 19: 514-6.

20. Cali A, Orenstein J M, Kotler DP et al. A comparison of two microsporidian parasites in enterocytes of AIDS patients with chronic diarrhea. J Protozool 1991; 38: 965-85.

21. Cali A, Kotler DP, Orenstein J M. Septata intestinal is N. G., N. Sp., an intestinal microsporidian associated with chronic diarrhea and dissemination in AIDS patients. J Euk Microbiol 1993; 40: 101-12.

22. Canning EU, Hollister WS. Enterocytozoon bieneusi (Microspora): prevalence and pathogenicity in AIDS patients. Trans R Soc Trop Med Hyg 1990; 84: 181-6.

23. Ledford DK, Overman MD, Gonzalvo A et al. Microsporidiosis myositis in a patient with the acquired immunodeficiency syndrome. Ann Intern Med 1985; 102: 628-30.

24. Margileth AM, Strano AJ, Chandra $R$ et al. Disseminated nosematosis in an immunol ogi cally compromi sed infant. Arch Pathol 1973; 95: 145-50.

25. Shadduck J A, Greeley E. Microsporidia and human infections. Clin Microbiol Rev 1989; 2: 158-69.

26. Shadduck J A, Meccoli RA, Davis R et al. First isolation of a microsporidian from a human patient. J Infect Dis 1990; 162: 773-6.

27. Rabeneck L, Gyorkey F, Genta RM et al. The role of Microsporidia in the pathogenesis of HIV-related chronic diarrhea. Ann Intern Med 1993; 119: 895-9.

28. Svenson J , MacLean E, Kokoskin-Nelson J et al. Microsporidiosis in AIDS patients. Can Comm Dis Rep 1993; 19: 13-5.

29. Blanshard C, Ellis DS, Tovey DG et al. Treatment of intestinal microsporidiosis with albendazole in patients with AIDS. AIDS 1992; 6: 311-3.

30. Dore GJ, Marriott DJ, Hing MC et al. Disseminated microsporidiosis due to Septata intestinalis in nine patients infected with the human immunodeficiency virus: response to therapy with al bendazol. Clin Infect Dis 1995; 21: 70-6.

31. Kelly P, McPhail G, N gwenya B. Septata intestinal is: a new microsporidian in Africa (letter). Lancet 1994; 344: 271-2.

32. Lecuit M, Oksenhendler E, Sarfati C. Use of albendazol for disseminated microsporidian infection in a patient with AIDS. Clin Infect Dis 1994; 19: 332-3.

33. Molina J M, Oksenhendler E, Beauvais B et al. Disseminated microsporidiosis due to Septata intestinalis in patients with AIDS: clinical features and response to al bendazole therapy. J Infect Dis 1995; 171: 245-9.

34. Orenstein J M, Dieterich DT, Kotler DP. Systemic dissemination by a newly recognized intestinal Microsporidia species in AIDS. AIDS 1992; 6: 1.143-50.

35. Orenstein J M, Tenner M, Cali A et al. A microsporidian previously undescribed in humans, infecting enterocytes and macrophages, and associated with diarrhea in an acquired immunodeficiency syndrome patient. Hum Pathol 1992; 23: 722-8.

36. Schwartz DA, Visvesvara GS, Diesenhouse MC et al. Pathologic features and immunofluorescent antibody demonstration of ocular microsporidiosis (Encephalitozoon hellem) in seven patients with acquired immunodeficiency syndrome [see comments]. Am J Ophthalmol 1993; 115: 285-92.

37. Weber $\mathrm{R}, \mathrm{K}$ uster $\mathrm{H}$, Visvesvara GS et al. Disseminated microsporidiosis due to Encephalitozoon hellem: pulmonary colonization, microhematuria, and mild conjunctivitis in a patient with AIDS. Clin Infect Dis 1993; 17: 415-9.

38. Weber R, Bryan RT. Microsporidial infections in immunodeficient and immunocompetent patients. Clin I nfect Dis 1994; 19: 517-21.

39. Avery SW, A.H.U. The isolation of microsporidia and other pathogens from concentrated ditch water.J Am M osq Control Assoc 1987; 3: 54-8.

40. Weber R, Kuster $\mathrm{H}$, Keller $\mathrm{R}$ et al. Pulmonary and intestinal microsporidiosis in a patient with the acquired immunodeficiency syndrome. Am Rev Respir Dis 1992; 146: 1.603-5.

41. Schwartz DA, Bryan RT, Hewan-Lowe KO et al. Disseminated microsporidiosis (Encephalitozoon hellem) and acquired immunodeficiency syndrome. Autopsy evidence for respiratory acquisition. Arch Pathol Lab Med 1992; 116: 660-8.

42. Hunt RD, King KW, Foster HL. Encephalitozoonosis: evidence for vertical transmission. J I nfect Dis 1972; 126: 212-4.

43. Orenstein J, Chiang J, Steinberg $W$ et al. Intestinal microsporidiosis as a cause of diarrhea in human immunodeficiency virus-infected patients: a report of 20 cases. Hum Pathol 1990; 21: 475-81.

44. Kotler DP, Francisco AF, Clayton $F$ et al. Small intestinal injury and parasitic diseases in AIDS. Ann Intern Med 1990; 113: 444-9.

45. Schattenkerk J KME, van Gool T, van Ketel RJ et al. Clinical significance of small-intestinal microsporidioses in HIV-1 infected individuals. Lancet 1991; 337: 895-8.

46. De Groote MA, Visvesvara G, Wilson ML. Polymerase chain reaction and culture confirmation of disseminated Encephalitozoon cuniculi in a patient with AIDS: successful therapy with albendazol. J Infect Dis 1995; 171: 1.375-8.

47. Bergquist NR, Stintzing $G$, Smedman $L$ et al. Diagnosis of encephalitozoonosis in man by serological tests. $\mathrm{Br}$ Med J 1984; 288: 902.

48. Macher AM, NeafieR, Angritt $P$ et al. Microsporidial myositis and the AIDS: a four year follow-up. 1988; Ann Intern Med 109: 343 [letter].

49. Ashton N, Wirasinha PA. Encephalitozoonosis (nosematosis) of the cornea. Br J Ophthalmol 1973; 57: 669-74.

50. Cali A, Meisler DM, Lowder CL. Corneal microsporidiosis: characterization and identification. J Protozool 1991; 38: 215S-217S.

51. Schwartz DA, Visvesvara GS, Leitch GJ et al. Pathology of symptomatic microsporidial (Encephalitozoon hellem) bronchiolitis in the acquired immunodeficiency syndrome: a new respiratory pathogen diagnosed from lung biopsy, bronchoalveolar lavage, sputum, and tissue culture [see comments]. Hum Pathol 1993; 24: 937-43.

52. Terada S, Reddy R, J effers $L$ J et al. Microsporidian hepatitis in the acquired immunodeficiency syndrome. Ann I ntern Med 1987; 107: 61-2.

53. Zender HO, Arrigoni E, Eckert J et al. A case of Encephalitozoon cuniculi peritonitis in a patient with AIDS. Am J Clin Pathol 1989; 92: 352-6.

54. Chupp GL, Alroy J, Adelman LS et al. Myositis due to pleistophora (microsporidia) in a patient with AIDS. Clin Infect Dis 1993; 16: 15-21.

55. Kotler DP, Gaetz HP, Lange M et al. Enteropathy associated with the acquired immunodeficiency syndrome. Ann Intern Med 1984; 101: 421-7.

56. Asmuth DM, Degirolami PC, Federmann M et al. Clinical features of microsporidiosis in patients with AIDS. Clin I nfect Dis 1993; 18: 819-25.

57. Bouchaud O, Houzé S, Saada M et al. Intestinal microsporidiosis in AIDS patients: high percentage of asymptomatic 
carriers. $9^{\text {th }}$ International Conference of AIDS, 1993; 1: 387.

58. Molina J M, Sarfati C, Beauvais B et al. Intestinal microsporidiosis in human immunodeficiency virus-infected patients with chronic unexplained diarrhea: prevalence and clinical and biologic features. J Infect Dis 1993; 167: 217-21.

59. Orenstein J M. Microsporidiosis in the acquired immunodeficiency syndrome. J Parasitol 1991; 77: 843-64.

60. Weber R, Muller A, Spycher MA et al. Intestinal Enterocytozoon bieneusi microsporidiosis in an HIV-infected patient: diagnosis by ileo-colonoscopic biopsies and long-term follow up. Clin Invest 1992; 70: 1.019-23.

61. Eeftinck Schattenkerk J KM, Van Gool T, Van Ketel RJ . Clinical significance of small-intestinal microsporidiosis in HIV-1-infected individuals. Lancet 1991; 337: 895-8.

62. Beaugerie L, Teilhac MF, Deluol AM et al. Cholangiopathy associated with microsporidia infection of the common bile duct mucosa in a patient with HIV infection. Ann Intern Med 1992; 117: 401-2.

63. McWhinney PH, Nathwani D, Green ST et al. Microsporidiosis detected in association with AIDS-related sclerosing cholangitis [letter]. AIDS 1991; 5: 1.394-5.

64. Pol S, Romana CA, Richard S et al. Microsporidia infection in patients with the human immunodeficiency virus and unexplained cholangitis. N Engl J Med 1993; 328: 95-9.

65. Canning EU, Curry A, Lacey CJ et al. Ultrastructure of Encephalitozoon sp. infecting the conjunctiva, corneal and nasal epithelia of a patient with AIDS. Eur J Protistol 1992; 28: 226-37.

66. Lacey CJ N, Clark A, Frazer P et al. Chronic microsporidian infection in thenasal mucosae, sinuses and conjuntivaein HIV disease. Gen U rinary Med 1992; 68: 179-81.

67. Schwartz DA, Cali A, Visvesvara GS et al. A nasal microsporidian with unusual features from a patient with AIDS. $9^{\text {th }}$ International Conference of AIDS, 1993; 1: 384.

68. WankeCA, Mattia AR. A 36-year-ol d man with AIDS, increase in chronic diarrhea, and intermittent fever and chills. N E ngl J Med 1993; 329: 1.946-54.

69. Schwartz DA, Visvesvara GS, Weber $R$ et al. Male genital tract microsporidiosis and AIDS: prostatic abscess due to Encephalitozoon hellem. J Eukaryot Microbiol 1995; 61S.

70. Diesenhouse MC, Wilson LA, Corrent GF et al. Treatment of microsporidial keratoconjunctivitis with topical fumagillin [see comments]. Am J Ophthalmol 1993; 115: 293-8.

71. CDC. Microsporidian keratoconjunctivitis in patients with AIDS. MMWR 1990; 39: 188-9.

72. Gunarsson G, Hurlbut D, DeGirolami PC et al. Multiorgan microsporidiosis: report of five cases and review. Clin Infect Dis 1995; 21: 37-44.

73. Kotler DP, Giang TT, Garro ML et al. Light microscopic diagnosis of microsporidiosis in patients with AIDS. Am J Gastroenterol 1994; 89: 540-4.

74. Weber R, Bryan RT, Owen RL et al. Improved light-microscopical detection of microsporidia spores in stool and duodenal aspirates. N Engl J Med 1992; 326: 161-6.

75. Van Gool T, Snijders F, Reiss P et al. Diagnosis of intestinal and disseminated microsporidial infections in patients with $\mathrm{HIV}$ by a new rapid fluorescencetechnique. J Clin Pathol 1993; 46: 694-9.

76. Zierdt CH, Gill VJ, Zierdt WS. Detection of microsporidian spores in clinical samples by indirect fluorescent antibody assay using whole-cell antisera to Encephalitozoon cuniculi and Encephalitozoon hellem. J Clin Microbiol 1993; 31: 3.071-4.

77. Van Gool T, Hollister WS, E eftinck Schattenkerk J KM et al. Diagnosis of Enterocytozoon bieneusi microsporidiosis in AIDS patients by recovery of spores from feces. Lancet 1990; 336: 697-8.

78. Orenstein J M, Tenner M, Kotler DP. Localization of infection by the microsporidian Enterocytozoon bieneusi in the gastrointestinal tract of AIDS patients with diarrhea. AIDS 1992; 6: 195-7.

79. Dieterich DT, Rahmin M. Cytomegalovirus colitis in AIDS: presentation in 44 patients and a review of the literature. J Acquired I mmune Defic Syndr 1991; 4(suppl. 1): S29-S35.

80. Katznelson $\mathrm{H}$, J amieson CA. Control of Nosema disease of honey bees with fumagillin. Science 1952; 115: 70-1.

81. Shadduck J A. Effect of fumagillin on in vitro multiplication of Encephalitozoon cuniculi. J Protozool 1980; 27: 202.

82. Dieterich DT, Lew EA, Kotler DP et al. Treatment with albendazol for intestinal disease due to Enterocytozoon bieneusi in patients with AIDS. J Infect Dis 1994; 169: 178-83.

83. Cello J P, Grendell J H, Basuk P et al. Effect of octreotide on refratory AIDS-associated diarrhea. Ann Intern Med 1991; 115: 705-10.

84. Simon D, Weiss LM, Tanowitz HB et al. Light microscopic diagnosis of human microsporidiosis and variable response to octreotide. Gastroenterology 1991; 100: 271-3.

85. Wuhib T, Silva TMJ , Newman RD. Cryptosporidial and microsporidial infections in human immunodeficiency virus-infected patients in northeastern Brazil. J I nfect Dis 1994; 170: 494-7.

86. Muccioli C, Belfort J r. R, Guidugli T et al. Ceratoconjuntivite por microsporídeo em AIDS: descrição do primeiro caso brasileiro e revisão da literatura. Arq Bras Oftalm 1993; 56: 289-90. 